$\underline{\text { Preprint typeset in JHEP style. - HYPER VERSION }}$

MIT-CTP-3203

hep-th/0110204

\title{
Some exact results on the matter star-product in the half-string formalism
}

\author{
Nicolas Moeller \\ Center for Theoretical Physics, \\ Massachusetts Institute of Technology, \\ Cambridge, MA 02139, USA \\ moeller@mit.edu
}

\begin{abstract}
We show that the D25 sliver wavefunction, just as the D-instanton sliver, factorizes when expressed in terms of half-string coordinates. We also calculate analytically the star-product of two zero-momentum eigenstates of $\hat{x}$ using the vertex in the oscillator basis, thereby showing that the star-product in the matter sector can indeed be seen as multiplication of matrices acting on the space of functionals of half strings. We then use the above results to establish that the matrices $\rho_{1,2}$, conjectured by Rastelli, Sen and Zwiebach to be left and right projectors on the sliver, are indeed so.
\end{abstract}

KEYwords: Open String Field Theory, Half-Strings, Star-Product. 


\section{Contents}

1. Introduction 1

2. Factorization of the sliver 3

2.1 Notations 3

2.2 Proof of the factorization

3. Star-product in the configuration basis 9

3.1 An application: Left and right projectors on the sliver 9

3.2 Setting up the calculation 12

4. Technical details of the calculation 15

4.1 Term quadratic in $a^{\dagger} \quad 15$

$\begin{array}{lll}4.2 & \text { The delta function } & 17\end{array}$

\begin{tabular}{lll}
4.3 & Regular quadratic term in $x$ & 20 \\
\hline
\end{tabular}

4.4 Term linear in $a^{\dagger} \quad 21$

\begin{tabular}{ll}
\hline 4.5 & The overall normalization \\
\hline
\end{tabular}

5. Summary 24

\begin{tabular}{lll}
5.1 & Factorization of the sliver & 24 \\
\hline
\end{tabular}

\begin{tabular}{lll}
5.2 & Star-product & 25 \\
\hline 5.3 & Projectors on thesliver
\end{tabular}

\begin{tabular}{lll}
5.3 & Projectors on the sliver & 25 \\
\hline
\end{tabular}

6. Discussion and Conclusions 25

\section{Introduction}

It has early been proposed [1] that the Open String Field Theory star-product may be seen as an infinite dimensional local matrix algebra. Namely, if one writes a string functional $\psi$ as a functional $\psi\left(x_{\text {mid }}, x^{L}, x^{R}\right)$ of the string midpoint $x_{\text {mid }}$, the left-half modes $x^{L}$ and the right-half modes $x^{R}$, the star-product of two string functionals sharing the same midpoint could then be written

$$
\left(\psi_{1} \star \psi_{2}\right)\left(x_{\mathrm{mid}}, x^{L}, x^{R}\right)=\int[d y] \psi_{1}\left(x_{\mathrm{mid}}, x^{L}, y\right) \psi_{2}\left(x_{\mathrm{mid}}, y, x^{R}\right),
$$

where $y$ is a half-string coordinate. 
This operator formulation of the star-product had an early important application. Indeed, it was implicitly used by Gross and Jevicki in [2] and [3] to deduce the form of the star-product in terms of Neumann coefficients; it actually provided expressions of these Neumann coefficients without using the conformal mapping of the vertex. One of the results of the present paper is to take the reverse path: starting from the star-product given in terms of Neumann coefficients, we are able to show analytically that, in the zero-momentum matter sector, the star-product indeed has the form (1.1). For this, we carefully calculate the star-product $\left|x_{1}\right\rangle \star\left|x_{2}\right\rangle$ of two zero-momentum eigenstates of $\hat{x}$ with vanishing zero-mode $\left(x_{1,2}\right)_{0}=0$. We find that the result is proportional to $\delta\left(x_{1}^{R}-x_{2}^{L}\right)\left|x_{3}\right\rangle$, where the left-half of $x_{3}$ is equal to the left-half of $x_{1}$ and the right-half of $x_{3}$ is equal to the right-half of $x_{2}$ (namely $x_{3}^{L}=x_{1}^{L}$ and $x_{3}^{R}=x_{2}^{R}$ ). The delta function implies that the result is zero except when the right-half of $x_{1}$ coincides with the left-half of $x_{2}$. This calculation is technical and will, moreover, require the use of a regularization scheme. At last, using the fact that the states $|x\rangle$ form a complete basis of zero-momentum states, we can formally derive the form of the star-product (1.1) between zero-momentum string wavefunctions.

Early attempts have been made to develop the operator formalism of the star-product ([6] - [9]). And more recently, this formalism gained new importance in the context of Vacuum String Field Theory, proposed by Rastelli, Sen and Zwiebach in ([10] - [13]) (see [15] for a nice review). In this proposal, the BRST operator around the stable vacuum is taken to be pure ghost, and thus the equation of motion around the nonperturbative vacuum factorizes into a matter part and a ghost part. In particular in the matter part, the equation of motion takes the form of a projection equation:

$$
\psi_{\mathrm{m}} \star \psi_{\mathrm{m}}=\psi_{\mathrm{m}}
$$

The simplest nontrivial zero-momentum solution of this equation, besides the identity, is the renowned sliver, which was first constructed in its geometric form by Rastelli and Zwiebach in [16] as the limit $n \rightarrow \infty$ of the wedge states $|n\rangle$. Kostelecky and Potting [17] later found an algebraic solution of (1.2), which was then conjectured by Rastelli, Sen and Zwiebach [11] to be the same as the geometric sliver. A nice and almost complete proof of this conjecture was given recently by Furuuchi and Okuyamain in [18]. It was also conjectured in [11] that the sliver solution corresponds to a space-filling D-25 brane, bringing us back from the stable vacuum to the perturbative vacuum.

An interesting property of the sliver was proposed in [12]: The authors found numerical evidence that, when expressed in terms of half-string modes, the sliver wavefunction seems to factorize into a left part and a right part. Their motivation was that, when the sliver is interpreted as the wedge state $|\infty\rangle$, the surface describing the sliver state is seen, in a suitable coordinate system, to be cut in two disjoint pieces: one corresponding to the left degrees of freedom and one corresponding to the right degrees of freedom (see [13 for a detailed treatment of the geometric picture). Gross and Taylor [19] independently found a similar property regarding the D-instanton sliver (a sliver localized in all 26 dimensions), and they were able to prove it. Their proof is based on the use of the overlap equations satisfied by the Neumann coefficients. These equations were formally shown to hold in ([2], [3]) for the Neumann coefficients found from the conformal mapping of the vertex, and they were interpreted as the condition that the vertex glues the right-half of the $r^{\text {th }}$ string to 
the left-half of the $(r+1)^{\text {th }}$ string. These equations thus implicitly contain information about the half-string formalism, which is necessary for the proof.

The proof that the D-instanton sliver wavefunction factorizes however doesn't immediately generalize to the sliver. It is one of the aims of this paper to present a proof of the factorization of the sliver. It is mainly based on the proof of Gross and Taylor, but requires some modifications due to the nontrivial transformations of the Neumann coefficients when going from the zero-momentum basis to the momentum-dependent basis. This factorization property is important in the projection operator techniques used in [12] and [19]. Indeed, if we write the sliver wavefunction as $\psi_{\Xi}\left(x^{L}, x^{R}\right)=f\left(x^{L}\right) f^{*}\left(x^{R}\right)$, we see that it is a rank one projector on the space of half-string functionals.

Finally, we use our previous results to show that the operators $\rho_{1,2}$ introduced by Rastelli, Sen and Zwiebach, satisfy some equations which allow us to interpret them as left and right projectors on the sliver, as conjectured in [12].

The outline of the paper is as follows. In section 2 we introduce our notation and prove that the sliver factorizes. In the course of the proof, we will derive several useful equations that will be used in section 4 . In section 3 , we set up the calculation of $\left|x_{1}\right\rangle \star\left|x_{2}\right\rangle$ and we motivate it by giving an application of its result: we show that the operators $\rho_{1}$ and $\rho_{2}$ of [12] are indeed left and right projectors on the sliver. In section 4 , we complete the proof started in section 3 . Then in section 5 we collect some useful results established in this paper. And finally, in section 6, we discuss our results and propose further directions of investigation.

\section{Factorization of the sliver}

In [19], the authors proved that the D-instanton sliver factorizes when its wavefunction is expressed in terms of half-string modes. Using a similar proof, we show here that the sliver (that we sometimes call the D-25-brane sliver to avoid confusion) constructed in [11], factorizes as well.

\subsection{Notations}

Our notations are taken from different sources (in particular [11, [19, [18]). And as the same symbols are sometimes used for different quantities in different papers, we start this section by giving an exhaustive list of our own notation.

\section{Neumann coefficients}

The star-product of two states $|A\rangle$ and $|B\rangle$ is calculated as

$$
|A\rangle \star|B\rangle_{3}={ }_{1}\left\langle\left. A\right|_{2}\left\langle B \mid V_{3}\right\rangle_{123}\right.
$$

where $\left|V_{3}\right\rangle$ is the three-string vertex. We can write it explicitly in terms of Neumann coefficients, which will depend on the basis we choose. In the following, we will use two different bases: The 
zero-momentum basis, in which

$$
\left|V_{3}\right\rangle_{123}=\exp \left(-\frac{1}{2} \sum_{\substack{r, s \\ m, n \geq 1}} a_{m}^{(r) \dagger} \cdot V_{m n}^{r s} a_{n}^{(s) \dagger}\right)|0\rangle_{123}
$$

where the state $|0\rangle$ (the zero-momentum vacuum whose zero-mode part has been dropped) is annihilated by the $a_{n}$ 's for $n \geq 1$, and satisfies $\langle 0 \mid 0\rangle=1$. Note that a summation over Lorentz indices is implicit in the product. The $V^{r s}$ are the Neumann coefficients and the superscripts $r$ and $s$, labeling the Hilbert space, go from one to three. Note also that the modes $m, n$ run from one to infinity, and the states $|A\rangle$ and $|B\rangle$ star-multiplied using this vertex, must have zero momentum.

We will also use our momentum-dependent basis, in which

$$
\left|V_{3}\right\rangle_{123}=\exp \left(-\frac{1}{2} \sum_{\substack{r, s \\ m, n \geq 0}} a_{m}^{(r) \dagger} \cdot \tilde{\mathcal{V}}_{m n}^{r s} a_{n}^{(s) \dagger}\right)|\Omega\rangle_{123}
$$

where $a_{0}=\frac{1}{2}\left(p_{0}-2 i x_{0}\right)$ obeys the commutation relation $\left[a_{0}, a_{0}^{\dagger}\right]=1$, and the vacuum $|\Omega\rangle$, satisfying $\langle\Omega \mid \Omega\rangle=1$, is annihilated by all the $a_{n}$ 's including $a_{0}$, it is therefore different from $|0\rangle$. Note also that here the modes $m$ and $n$ run from zero to infinity, and the states $|A\rangle$ and $|B\rangle$ star-multiplied in this basis are general. This particular momentum-dependent basis was used in 19 to construct the D-instanton sliver, and the zero-momentum basis was earlier used to describe the sliver ([17], [11]).

It is time for some clarification: Matrices describing the D-instanton sliver have indices running from 0 to $\infty$, but the indices of the matrices used for the D-25-sliver run only from 1 to $\infty$. For clarity we will thus denote matrices whose indices start from zero with a ${ }^{\sim}$ such as $\tilde{M}$, as matrices without a ${ }^{\sim}$ will have indices starting from one. If a matrix $\tilde{M}$ is defined, we will always assume that the corresponding matrix $M$ is obtained from $\tilde{M}$ by deleting its first column and its first row. Moreover, the matrices transforming non-trivially from one basis to the other (like $V^{r s}$ ) will be written in calligraphic form in the momentum-dependent basis, and in roman form in the zeromomentum basis.

The matrices $V^{r s}\left(\tilde{\mathcal{V}}^{r s}\right)$ satisfy the following transposition and cyclicity properties:

$$
\left(V^{r s}\right)^{T}=V^{s r}, \quad V^{r s}=V^{(r+1)(s+1)} .
$$

They can be written in terms of a single matrix $U(\tilde{\mathcal{U}})$ :

$$
V^{r s}=\frac{1}{3}\left(C+\omega^{s-r} U+\omega^{r-s} \bar{U}\right), \quad \tilde{\mathcal{V}}^{r s}=\frac{1}{3}\left(\tilde{C}+\omega^{s-r} \tilde{\mathcal{U}}+\omega^{r-s} \overline{\tilde{\mathcal{U}}}\right)
$$

where $\tilde{C}_{m n}=(-1)^{m} \delta_{m n}$ and $\omega=e^{2 i \pi / 3}$. The matrices $U$ and $\tilde{\mathcal{U}}$ obey the following relations:

$$
\bar{U} \equiv U^{*}=C U C, \quad U^{2}=\bar{U}^{2}=1, \quad U^{\dagger}=U, \quad \bar{U}^{\dagger}=\bar{U},
$$


as well as similar equations for $\tilde{\mathcal{U}}$. The relation between $U$ and $\tilde{\mathcal{U}}$ is given by ([17], [11])

$$
U_{m n}=\tilde{\mathcal{U}}_{m n}+\frac{\tilde{\mathcal{U}}_{m 0} \tilde{\mathcal{U}}_{0 n}}{1-\tilde{\mathcal{U}}_{00}}
$$

We now define the matrices $X, Y$ and $Z$ :

$$
X=C V^{11}, \quad Y=C V^{12}, \quad Z=C V^{21} .
$$

They are symmetric and satisfy the relations:

$$
\begin{aligned}
& X+Y+Z=1, \\
& X^{2}+Y^{2}+Z^{2}=1, \\
& Y Z=X^{2}-X, \\
& C X C=X, \quad C Y C=Z, \quad C Z C=Y .
\end{aligned}
$$

\section{Half-string formalism}

Let us now review the half-string formalism as it was presented in [12]. Let us first write the mode expansion of the open string coordinate:

$$
X^{\mu}(\sigma)=x_{0}^{\mu}+\sqrt{2} \sum_{n=1}^{\infty} x_{n}^{\mu} \cos (n \sigma), \quad 0 \leq \sigma \leq \pi .
$$

We then define the left and right half of the string as:

$$
\begin{aligned}
& X^{L \mu}(\sigma)=X^{\mu}(\sigma / 2)-X^{\mu}(\pi / 2), \quad 0 \leq \sigma \leq \pi, \\
& X^{R \mu}(\sigma)=X^{\mu}(\pi-\sigma / 2)-X^{\mu}(\pi / 2), \quad 0 \leq \sigma \leq \pi .
\end{aligned}
$$

They have the following mode expansion:

$$
X^{L(R) \mu}(\sigma)=\sqrt{2} \sum_{n=1}^{\infty} x_{n}^{L(R) \mu} \cos \left(\left(n-\frac{1}{2}\right) \sigma\right) .
$$

We can express the relation between the full modes and the half modes in terms of matrices $A^{ \pm}$ (from now on, we will not write the spacetime indices, which will be implicit):

$$
x=A^{+} x^{L}+A^{-} x^{R},
$$

where

$$
A_{n m}^{ \pm}= \pm \frac{1}{2} \delta_{n, 2 m-1}+\frac{1}{2 \pi}\left(1+(-1)^{n}\right)(-1)^{m+\frac{1}{2} n-1}\left(\frac{1}{2 m+n-1}+\frac{1}{2 m-n-1}\right) .
$$

We can invert the relation (2.13), and we get:

$$
x^{L}=\tilde{A}^{+} x, \quad x^{R}=\tilde{A}^{-} x,
$$


where $^{1}$

$$
\tilde{A}_{m n}^{ \pm}=2 A_{n m}^{ \pm}-\frac{1}{\pi}\left(1+(-1)^{n}\right)(-1)^{m+\frac{1}{2} n-1} \frac{2}{2 m-1} .
$$

Let us note the following useful relations:

$$
A^{+} \tilde{A}^{+}+A^{-} \tilde{A}^{-}=1, \quad \tilde{A}^{ \pm} A^{\mp}=0, \quad \tilde{A}^{ \pm} A^{ \pm}=1,
$$

as well as

$$
A^{+}=C A^{-}, \quad \tilde{A}^{+}=\tilde{A}^{-} C .
$$

It will be useful to use the matrix $\tilde{\Upsilon}$, defined by ${ }^{2}$

$$
\begin{aligned}
\tilde{\Upsilon}_{2 k+1,2 n} & =\tilde{\Upsilon}_{2 n, 2 k+1}=\frac{4(-1)^{k+n}(2 k+1)}{\pi\left((2 k+1)^{2}-4 n^{2}\right)} \quad(n \neq 0), \\
\tilde{\Upsilon}_{2 k+1,0} & =\tilde{\Upsilon}_{0,2 k+1}=\frac{2 \sqrt{2}(-1)^{k}}{\pi(2 k+1)},
\end{aligned}
$$

all other elements being zero. We can write a relation between $A^{ \pm}$and $\Upsilon$ :

$$
A_{n m}^{ \pm}= \pm \frac{1}{2} \delta_{n, 2 m-1}+\frac{1}{2} \Upsilon_{n, 2 m-1}, \quad n, m \geq 1 .
$$

Finally, we note the relation between the oscillators $a_{n}$ and the modes $x_{n}$ :

$$
\hat{x}=\frac{i}{2} E \cdot\left(a-a^{\dagger}\right)
$$

where $^{3}$

$$
\tilde{E}_{n m}=\delta_{n m} \sqrt{\frac{2}{n}}+\delta_{n 0} \delta_{m 0}
$$

\section{Parity notation}

We will adopt the idea, used in [19], to split matrices according to the parity of the indices. A matrix $M$ will be rewritten as $M=\left(\begin{array}{ll}M_{o o} & M_{o e} \\ M_{e o} & M_{e e}\end{array}\right)$, where the subscripts $o$ and $e$ mean odd and even respectively, to denote odd and even indices. With this notation, that we shall call parity notation, the matrix $\tilde{\Upsilon}$ can be rewritten $\tilde{\Upsilon}=\left(\begin{array}{cc}0 & \tilde{\Upsilon}_{o e} \\ \tilde{\Upsilon}_{e o} & 0\end{array}\right)$. From the definition (2.19), one can check that $\tilde{\Upsilon}_{o e} \tilde{\Upsilon}_{e o}=\tilde{\Upsilon}_{e o} \tilde{\Upsilon}_{o e}=1$, which implies that $\tilde{\Upsilon}^{2}=1$; when one truncates to matrices with nonzero indices only, $\Upsilon_{e o} \Upsilon_{o e}=1$ still holds, but now $\Upsilon_{o e} \Upsilon_{e o} \neq 1$. In other words, $\Upsilon_{e o}$ is a left inverse of $\Upsilon_{o e}$ but not a right inverse. This will have some implications in the way we will establish the factorization.

\footnotetext{
${ }^{1}$ Note that the relation between $A$ and $\tilde{A}$ has nothing to do with the notation introduced earlier; we want here to keep the notations of $[12$. The indices of both $A$ and $\tilde{A}$ run from one to infinity. We hope that the reader will not get confused with this slight abuse of notation.

${ }^{2}$ This matrix is called $X$ in [19], but here we keep the symbol $X$ for the matrix $X=C V^{11}$.

${ }^{3}$ Here we adopt the definition of [1], which differs from the one in [19] by a factor of $\sqrt{2}$.
} 
It will be useful in the following to know the form of $X, Y$ and $Z$ in the parity notation. From (2.5), we get:

$$
X=\frac{1}{3}\left(\begin{array}{cc}
1-2 U_{o o} & 0 \\
0 & 1+2 U_{e e}
\end{array}\right), Y=\frac{1}{3}\left(\begin{array}{cc}
1+U_{o o} & -\sqrt{3} i U_{o e} \\
\sqrt{3} i U_{e o} & 1-U_{e e}
\end{array}\right), Z=\frac{1}{3}\left(\begin{array}{cc}
1+U_{o o} & \sqrt{3} i U_{o e} \\
-\sqrt{3} i U_{e o} & 1-U_{e e}
\end{array}\right) .
$$

\section{The sliver}

The sliver state $|\Xi\rangle$ is given by

$$
|\Xi\rangle=\mathcal{N}^{26} \exp \left(-\frac{1}{2} a^{\dagger} \cdot S a^{\dagger}\right)|0\rangle
$$

where $\mathcal{N}$ is a normalization factor. And $S=C T$, with

$$
T=(2 X)^{-1}(1+X-\sqrt{(1+3 X)(1-X)}) .
$$

From the property $S=C S C$, we have that $S_{o e}=S_{e o}=0$.

\subsection{Proof of the factorization}

In [12], it was shown that the sliver wavefunction, when expressed in terms of half-string modes $x^{L}$ and $x^{R}$, is

$$
\psi_{\Xi}\left(x^{L}, x^{R}\right)=\langle x \mid \Xi\rangle=\tilde{\mathcal{N}}^{26} \exp \left(-\frac{1}{2} x^{L} \cdot K x^{L}-\frac{1}{2} x^{R} \cdot K x^{R}-x^{L} \cdot L x^{R}\right),
$$

where $\tilde{\mathcal{N}}^{26}$ is a normalization factor, $K=A^{+^{T}} V A^{+}=A^{-T} V A^{-}, L=A^{+T} V A^{-}$, and $V=$ $2 E^{-1} \frac{1-S}{1+S} E^{-1}$. By left-right factorization of the sliver we mean that the cross terms in the exponential must vanish. We thus want to show that

$$
L \equiv A^{+^{T}} V A^{-}=0
$$

To prove (2.27), we first note that (2.20) implies the following equivalent form of (2.27):

$$
V_{o o}=\Upsilon_{o e} V_{e e} \Upsilon_{e o},
$$

where we have used that $V_{o e}=V_{e o}=0$. Now we want to express $V$ in terms of $U$. This was done in [19], and their result still holds with our $U$. Namely, we have:

$$
V \equiv 2 E^{-1} \frac{1-S}{1+S} E^{-1}=2 E^{-1}\left(\begin{array}{cc}
\sqrt{3} \frac{\sqrt{1-U_{o o}}}{\sqrt{1+U_{o o}}} & 0 \\
0 & \frac{1}{\sqrt{3}} \frac{\sqrt{1-U_{e e}}}{\sqrt{1+U_{e e}}}
\end{array}\right) E^{-1},
$$

which together with (2.28) gives the factorization condition:

$$
3 \sqrt{\frac{1-U_{o o}}{1+U_{o o}}}=E \Upsilon_{o e} E^{-1} \sqrt{\frac{1-U_{e e}}{1+U_{e e}}} E^{-1} \Upsilon_{e o} E,
$$

which bears some similarities with the factorization condition of the D-instanton sliver [19]. Our goal now is to prove (2.29). 
For this, we first show that (2.29) will hold if the following two identities hold:

$$
\begin{gathered}
\sqrt{3} i U_{e o}\left(1+U_{o o}\right)^{-1}=E^{-1} \Upsilon_{e o} E, \\
-\sqrt{3} i\left(1-U_{o o}\right)\left(U_{e o}\right)^{-1}=E \Upsilon_{o e} E^{-1} .
\end{gathered}
$$

Indeed, using the fact that $U^{2}=1$ (2.6), we find:

$$
\left(\begin{array}{ll}
U_{o o} U_{o o}+U_{o e} U_{e o} & U_{o o} U_{o e}+U_{o e} U_{e e} \\
U_{e o} U_{o o}+U_{e e} U_{e o} & U_{e o} U_{o e}+U_{e e} U_{e e}
\end{array}\right)=\left(\begin{array}{ll}
1 & 0 \\
0 & 1
\end{array}\right) .
$$

From this, one has

$$
\begin{aligned}
& U_{e o}+U_{e e} U_{e o}+U_{e o} U_{o o}+U_{e e} U_{e o} U_{o o}=U_{e o}-U_{e e} U_{e o}-U_{e o} U_{o o}+U_{e e} U_{e o} U_{o o} \\
\Rightarrow & \left(1+U_{e e}\right) U_{e o}\left(1+U_{o o}\right)=\left(1-U_{e e}\right) U_{e o}\left(1-U_{o o}\right) \\
\Rightarrow & \frac{1+U_{o o}}{1-U_{o o}}=\left(U_{e o}\right)^{-1} \frac{1-U_{e e}}{1+U_{e e}} U_{e o} \\
\Rightarrow & \sqrt{\frac{1+U_{o o}}{1-U_{o o}}}=\left(U_{e o}\right)^{-1} \sqrt{\frac{1-U_{e e}}{1+U_{e e}}} U_{e o} \\
\Rightarrow & \sqrt{\frac{1-U_{o o}}{1+U_{o o}}}=\left(1-U_{o o}\right)\left(U_{e o}\right)^{-1} \sqrt{\frac{1-U_{e e}}{1+U_{e e}}} U_{e o} \frac{1}{1+U_{o o}},
\end{aligned}
$$

where in the fourth line, we assumed that the square root is defined by its power expansion. Now substituting (2.30) and (2.31) into (2.33) we get exactly (2.29).

We will now prove the identities (2.30) and (2.31). Let us rewrite four equations that were established in [19] from the overlap equations:

$$
\begin{array}{r}
\left(1+\tilde{\mathcal{U}}_{o o}\right)-\sqrt{3} i \tilde{E}^{-1} \tilde{\Upsilon}_{o e} \tilde{E} \tilde{\mathcal{U}}_{e o}=0, \\
-\sqrt{3} i \tilde{E}^{-1} \tilde{\Upsilon}_{o e} \tilde{E}\left(1+\tilde{\mathcal{U}}_{e e}\right)+\tilde{\mathcal{U}}_{o e}=0, \\
3\left(1-\tilde{\mathcal{U}}_{o o}\right)-\sqrt{3} i \tilde{E} \tilde{\Upsilon}_{o e} \tilde{E}^{-1} \tilde{\mathcal{U}}_{e o}=0, \\
\sqrt{3} i \tilde{E} \tilde{\Upsilon}_{o e} \tilde{E}^{-1}\left(1-\tilde{\mathcal{U}}_{e e}\right)-3 \tilde{\mathcal{U}}_{o e}=0 .
\end{array}
$$

Multiplying (2.34) by $\tilde{\Upsilon}_{e o} \tilde{E}$ on the left, one gets

$$
\tilde{\Upsilon}_{e o} \tilde{E}\left(1+\tilde{\mathcal{U}}_{o o}\right)-\sqrt{3} i \tilde{E} \tilde{\mathcal{U}}_{e o}=0 .
$$

Our claim is that this equation still holds after replacing $\mathcal{U}$ by $U$ and truncating to nonzero indices:

$$
\Upsilon_{e o} E\left(1+U_{o o}\right)-\sqrt{3} i E U_{e o}=0 .
$$


To prove this, let us evaluate the $(2 n, 2 m-1)$ component of the lhs of (2.39), for $n, m \geq 1$ :

$$
\begin{aligned}
& \Upsilon_{2 n, 2 k-1} \frac{1}{\sqrt{2 k-1}}\left(\delta_{2 k-1,2 m-1}+U_{2 k-1,2 m-1}\right)-\sqrt{3} i \frac{1}{\sqrt{2 n}} U_{2 n, 2 m-1} \\
= & \Upsilon_{2 n, 2 k-1} \frac{1}{\sqrt{2 k-1}}\left(\delta_{2 k-1,2 m-1}+\tilde{\mathcal{U}}_{2 k-1,2 m-1}\right)-\sqrt{3} i \frac{1}{\sqrt{2 n}} \tilde{\mathcal{U}}_{2 n, 2 m-1}+ \\
& +\left(\Upsilon_{2 n, 2 k-1} \frac{1}{\sqrt{2 k-1}} \tilde{\mathcal{U}}_{2 k-1,0}-\sqrt{3} i \frac{1}{\sqrt{2 n}} \tilde{\mathcal{U}}_{2 n, 0}\right) \frac{\tilde{\mathcal{U}}_{0,2 m-1}}{1-\tilde{\mathcal{U}}_{00}} \\
= & {\left[\tilde{\Upsilon}_{e o} \tilde{E} \tilde{\mathcal{U}}_{o e}-\sqrt{3} i \tilde{E} \tilde{\mathcal{U}}_{e e}\right]_{2 n, 0} \frac{\tilde{\mathcal{U}}_{0,2 m-1}}{1-\tilde{\mathcal{U}}_{00}} } \\
= & {\left[\tilde{\Upsilon}_{e o} \tilde{E} \tilde{\mathcal{U}}_{o e}-\sqrt{3} i \tilde{E}\left(1+\tilde{\mathcal{U}}_{e e}\right)\right]_{2 n, 0} \frac{\tilde{\mathcal{U}}_{0,2 m-1}}{1-\tilde{\mathcal{U}}_{00}} } \\
= & 0,
\end{aligned}
$$

where in the first step we used (2.7), in the second step we used (2.38), and in the third step we introduced an identity matrix which gives no contribution because $E$ is diagonal and $n$ is $\geq 1$. And in the last line we used (2.35). It is straightforward to show that (2.39) implies (2.30).

Similarly, starting form (2.36) and using (2.37), one can prove (2.31) if we assume that the matrix $U_{e o}$ is invertible. This concludes our proof that the sliver wavefunction factorizes.

\section{Star-product in the configuration basis}

Here we want to calculate explicitly the star-product $\left|x_{1}\right\rangle \star\left|x_{2}\right\rangle$, where $\left|x_{1}\right\rangle$ and $\left|x_{2}\right\rangle$ are zeromomentum eigenstates of $\hat{x}$. Let us already write down what we expect to find:

$$
\left|x_{1}\right\rangle \star\left|x_{2}\right\rangle \sim \delta\left(x_{2}^{L}-x_{1}^{R}\right)\left|x_{3}\right\rangle
$$

where the left-half of $x_{3}$ is the left-half of $x_{1}$ and the right-half of $x_{3}$ is the right-half of $x_{2}$, namely

$$
x_{3}=A^{+} x_{1}^{L}+A^{-} x_{2}^{R} .
$$

The delta function is there because we expect the star-product to be nonzero only when the righthalf of $x_{1}$ coincides with the left-half of $x_{2}$. We will see that this delta function indeed arises after regularization of singular terms.

Before proving (3.1), we want to motivate its usefulness in the following subsection.

\subsection{An application: Left and right projectors on the sliver}

In [12], the authors defined a set of projectors ${ }^{4} \rho_{1}$ and $\rho_{2}$ :

$$
\rho_{1}=\frac{Z+T Y}{(1+T)(1-X)}, \quad \rho_{2}=\frac{Y+T Z}{(1+T)(1-X)},
$$

\footnotetext{
${ }^{4}$ The notation of [12] is a little different of that in [11] that we are using here: their $Y$ is our $Z$ and their $Z$ is our $Y$. Moreover their expressions for the projectors can be simplified by use of the relation: $-X Y+Y^{2}=Z$ (and similarly: $\left.-X Z+Z^{2}=Y\right)$.
} 
satisfying

$$
\rho_{1}^{2}=\rho_{1}, \quad \rho_{2}^{2}=\rho_{2}, \quad \rho_{1} \rho_{2}=\rho_{2} \rho_{1}=0 .
$$

They form a complete set of symmetric projectors:

$$
\rho_{1}^{T}=\rho_{1}, \quad \rho_{2}^{T}=\rho_{2}, \quad \rho_{1}+\rho_{2}=1 .
$$

We also note the following relations:

$$
C \rho_{1} C=\rho_{2}, \quad \rho_{1}-\rho_{2}=\frac{Z-Y}{\sqrt{(1-X)(1+3 X)}} .
$$

The authors of [12] conjectured that $\rho_{1}$ satisfies

$$
\rho_{1}(1+S) E \tilde{A}^{+T}=0,
$$

which also implies that $\rho_{2}(1+S) E \tilde{A}^{-T}=0$. Equation 3.7 means that $\rho_{1}$ is a left projector on the sliver in the following sense ${ }^{5}$ : Given a vector $\beta$, the state $\left(\beta \cdot a^{\dagger}\right)|\Xi\rangle$ can be written in terms of the left position operator $\hat{x}^{L}$ acted on the sliver:

$$
\forall \beta: \quad\left(\exists \lambda \text { such that }\left(\beta \cdot a^{\dagger}\right)|\Xi\rangle=\left(\lambda \cdot \hat{x}^{L}\right)|\Xi\rangle\right) \Leftrightarrow \rho_{1} \beta=0 .
$$

Let us show that (3.7) implies (3.8). We will do that in two steps: First we prove that (3.7) implies the " $\Rightarrow$ " part of (3.8). Indeed, assume that we can write $\left(\beta \cdot a^{\dagger}\right)|\Xi\rangle$ as $\left(\lambda \cdot \hat{x}^{L}\right)|\Xi\rangle$ for some $\lambda$, then:

$$
\begin{aligned}
\left(\lambda \cdot \hat{x}^{L}\right)|\Xi\rangle & =\left(\frac{i}{2} \lambda \cdot \tilde{A}^{+} E(1+S) a^{\dagger}\right)|\Xi\rangle \\
& =\left(\frac{i}{2}(1+S) E \tilde{A}^{+T} \lambda\right) \cdot a^{\dagger}|\Xi\rangle \\
& =\left(\beta \cdot a^{\dagger}\right)|\Xi\rangle,
\end{aligned}
$$

where $\beta=\frac{i}{2}(1+S) E \tilde{A}^{+T} \lambda$, and thus, from (3.7), $\rho_{1} \beta=0$.

Now let us prove that $(3.7)$ implies the " $\Leftarrow$ " part of (3.8). Remembering that $\tilde{A}^{+T} A^{+T}+$ $\tilde{A}^{-T} A^{-T}=1$, and assuming that $(1+S)$ is invertible, we can write:

$$
\beta=(1+S) E\left(\tilde{A}^{+T} A^{+T}+\tilde{A}^{-T} A^{-T}\right) \mu,
$$

for some vector $\mu$. Now $\rho_{1} \beta=0$ implies (from (3.7)) that $\rho_{1}(1+S) E \tilde{A}^{-T} A^{-T} \mu=0$. Therefore

$$
(1+S) E \tilde{A}^{-T} A^{-T} \mu=\left(\rho_{1}+\rho_{2}\right)(1+S) E \tilde{A}^{-T} A^{-T} \mu=\rho_{2}(1+S) E \tilde{A}^{-T} A^{-T} \mu=0,
$$

where we have used (3.7) in the last step. Putting this into (3.10), we see that

$$
\left(\beta \cdot a^{\dagger}\right)|\Xi\rangle=\left((1+S) E \tilde{A}^{+T} A^{+T} \mu \cdot a^{\dagger}\right)|\Xi\rangle=\frac{i}{2}\left((1+S) E \tilde{A}^{+T} \lambda \cdot a^{\dagger}\right)|\Xi\rangle=\left(\lambda \cdot \hat{x}^{L}\right)|\Xi\rangle,
$$

where we have defined $\lambda=-2 i A^{+T} \mu$. We thus have that (3.7) implies that $\rho_{1}$ is a left projector on the sliver in the sense of (3.8).

\footnotetext{
${ }^{5} \mathrm{I}$ thank B. Zwiebach for this definition
} 
We will now give a proof of (3.7) based on the factorization of the sliver and on the form of the star-product in the configuration basis (3.1). For that, we will show that (3.7) is equivalent to the vanishing of

$$
\left(\left(\lambda \cdot \hat{x}^{R}\right)|\Xi\rangle\right) \star|\Xi\rangle
$$

where $\lambda$ is a given vector of numbers.

Indeed, using that $a|\Xi\rangle=-S a^{\dagger}|\Xi\rangle$, one gets

$$
\begin{aligned}
\left(\lambda \cdot \hat{x}^{R}\right)|\Xi\rangle & =\left(\lambda \cdot \tilde{A}^{-} \hat{x}\right)|\Xi\rangle \\
& =\left(\frac{i}{2} \lambda \cdot \tilde{A}^{-} E\left(a-a^{\dagger}\right)\right)|\Xi\rangle \\
& =\left(-\frac{i}{2} \lambda \cdot \tilde{A}^{-} E(1+S) a^{\dagger}\right)|\Xi\rangle \\
& =\left(-\frac{i}{2} a^{\dagger} \cdot C(1+S) E \tilde{A}^{+T} \lambda\right)|\Xi\rangle
\end{aligned}
$$

where in the last line we used $\tilde{A}^{+}=\tilde{A}^{-} C$. Now recall that, in $\llbracket 12$, the authors proved that:

$$
\left(e^{-a^{\dagger} \cdot C \beta_{1}}|\Xi\rangle\right) \star\left(e^{-a^{\dagger} \cdot C \beta_{2}}|\Xi\rangle\right)=e^{-\mathcal{C}\left(\beta_{1}, \beta_{2}\right)} e^{-a^{\dagger} \cdot C\left(\rho_{1} \beta_{1}+\rho_{2} \beta_{2}\right)}|\Xi\rangle
$$

where

$$
\mathcal{C}\left(\beta_{1}, \beta_{2}\right)=\frac{1}{2}\left(\beta_{1}, \beta_{2}\right) \frac{C}{(1+T)(1-X)}\left(\begin{array}{cc}
X(1-T) & Z \\
Y & X(1-T)
\end{array}\right)\left(\begin{array}{l}
\beta_{1} \\
\beta_{2}
\end{array}\right),
$$

and $\beta_{1}$ and $\beta_{2}$ are vectors of numbers. Substituting $\beta_{1}=t \beta$, where $t$ is a real number, and $\beta_{2}=0$ into (3.15) and (3.16), one gets

$$
\left(e^{-a^{\dagger} \cdot C \beta t}|\Xi\rangle\right) \star|\Xi\rangle=e^{-\frac{1}{2} t^{2} \beta \cdot \frac{C X(1-T)}{(1+T)(1-X)} \beta} e^{-a^{\dagger} \cdot C \rho_{1} \beta t}|\Xi\rangle
$$

Differentiating this last equation with respect to $t$ and setting $t=0$ gives us

$$
\left(\left(-a^{\dagger} \cdot C \beta\right)|\Xi\rangle\right) \star|\Xi\rangle=\left(-a^{\dagger} \cdot C \rho_{1} \beta\right)|\Xi\rangle
$$

Now we substitute $\beta \equiv \frac{i}{2}(1+S) E \tilde{A}^{+T} \lambda$ into (3.18) and we use (3.14) to get

$$
\left(\left(\lambda \cdot \hat{x}^{R}\right)|\Xi\rangle\right) \star|\Xi\rangle=\left(-\frac{i}{2} a^{\dagger} \cdot C \rho_{1}(1+S) E \tilde{A}^{+T} \lambda\right)|\Xi\rangle
$$

Therefore, (3.7) holds if and only if (3.13) vanishes. 
We will now show that (3.13) indeed vanishes, by inserting two complete sets of zero-momentum states:

$$
\begin{aligned}
\left(\left(\lambda \cdot \hat{x}^{R}\right)|\Xi\rangle\right) \star|\Xi\rangle & =\left(\int\left[d x_{1}\right]\left|x_{1}\right\rangle\left\langle x_{1}\left|\left(\lambda \cdot \hat{x}^{R}\right)\right| \Xi\right\rangle\right) \star\left(\int\left[d x_{2}\right]\left|x_{2}\right\rangle\left\langle x_{2} \mid \Xi\right\rangle\right) \\
& =\left(\int\left[d x_{1}\right]\left|x_{1}\right\rangle\left\langle x_{1}\left|\left(\hat{x} \cdot \tilde{A}^{-T} \lambda\right)\right| \Xi\right\rangle\right) \star\left(\int\left[d x_{2}\right]\left|x_{2}\right\rangle\left\langle x_{2} \mid \Xi\right\rangle\right) \\
& =\int\left[d x_{1}\right]\left[d x_{2}\right]\left(x_{1} \cdot\left(\tilde{A}^{-}\right)^{T} \lambda\right) \psi_{\Xi}\left(x_{1}^{L}, x_{1}^{R}\right) \psi_{\Xi}\left(x_{2}^{L}, x_{2}^{R}\right)\left(\left|x_{1}\right\rangle \star\left|x_{2}\right\rangle\right) \\
& \sim \int\left[d x_{1}\right]\left[d x_{2}\right]\left(x_{1}^{R} \cdot \lambda\right) \psi_{\Xi}\left(x_{1}^{L}, x_{1}^{R}\right) \psi_{\Xi}\left(x_{2}^{L}, x_{2}^{R}\right) \delta\left(x_{1}^{R}-x_{2}^{L}\right)\left|A^{+} x_{1}^{L}+A^{-} x_{2}^{R}\right\rangle,
\end{aligned}
$$

where $\psi_{\Xi}\left(x^{L}, x^{R}\right)=\langle x \mid \Xi\rangle=\tilde{\mathcal{N}}^{26} \exp \left(-\frac{1}{2} x^{L} \cdot K x^{L}\right) \exp \left(-\frac{1}{2} x^{R} \cdot K x^{R}\right)$ is the sliver wavefunction. We will now factor the integral over $x_{1}$ into an integral over the left-half string and an integral over the right-half string: $\int\left[d x_{1}\right] \sim \int\left[d x_{1}^{L}\right]\left[d x_{1}^{R}\right]$, and focus on the piece of the integral(3.20) over $x_{1}^{R}$ only:

$$
\int\left[d x_{1}^{R}\right]\left(x_{1}^{R} \cdot \lambda\right) e^{-x_{1}^{R} \cdot K x_{1}^{R}}=0,
$$

because the integrand is odd. Therefore we have that

$$
\left(\left(\lambda \cdot \hat{x}^{R}\right)|\Xi\rangle\right) \star|\Xi\rangle=0
$$

And thus, (3.7) indeed holds.

\subsection{Setting up the calculation}

Before calculating the star-product $\left|x_{1}\right\rangle \star\left|x_{2}\right\rangle$, we shall make more precise what we mean by zeromomentum: we drop all dependence of the zero-mode $x_{0}$ by setting $\left(x_{1,2}\right)_{0}=0$, and our vacuum $|0\rangle$ is amputated from its zero-mode part (we can thus normalize it such that $\langle 0 \mid 0\rangle=1$ ). Let us now express $\left|x_{1}\right\rangle$ and $\left|x_{2}\right\rangle$ in the oscillator basis, namely ([12], 19]):

$$
\left|x_{1,2}\right\rangle=K_{0}^{26} \exp \left(-x_{1,2} \cdot E^{-2} x_{1,2}-2 i a^{\dagger} \cdot C E^{-1} x_{1,2}+\frac{1}{2} a^{\dagger} \cdot a^{\dagger}\right)|0\rangle
$$

where $K_{0}$ is a constant that can be calculated from the condition $\int[d x]|x\rangle\langle x|=1$, where the integration measure is defined as $[d x]=\prod_{n \geq 1} d x_{n}$. We get:

$$
K_{0}=\operatorname{det}\left(\left(\frac{2}{\pi}\right)^{\frac{1}{4}} E^{-\frac{1}{2}}\right)
$$

We introduce now, for reasons that will become clear below, the following regularized states:

$$
\left|x_{1,2}, \epsilon\right\rangle \equiv K_{0}^{26} \exp \left(-x_{1,2} \cdot E^{-2} x_{1,2}-2 i a^{\dagger} \cdot C E^{-1} x_{1,2}+\frac{1}{2}(1-\epsilon) a^{\dagger} \cdot a^{\dagger}\right)|0\rangle .
$$

We obviously have $\left|x_{1,2}\right\rangle=\lim _{\epsilon \rightarrow 0}\left|x_{1,2}, \epsilon\right\rangle$, but we will take the limit only at the end of our calculation because we will encounter singularities at $\epsilon=0$. 
The star-product is calculated using the vertex $\left|V_{3}\right\rangle$ in the oscillator basis:

$$
\left|x_{1}, \epsilon\right\rangle \star\left|x_{2}, \epsilon\right\rangle={ }_{1}\left\langle x_{1},\left.\epsilon\right|_{2}\left\langle x_{2}, \epsilon \mid V_{3}\right\rangle_{123},\right.
$$

where

$$
\left|V_{3}\right\rangle=\exp \left(-\frac{1}{2} \sum_{\substack{r, s \\ m, n \geq 1}} a_{m}^{(r) \dagger} \cdot V_{m n}^{r s} a_{n}^{(s) \dagger}\right)|0\rangle_{123} .
$$

A summation over Lorentz indices is implicit in the - product. Using the following formula ([17], [11):

$$
\begin{aligned}
& \left\langle 0\left|\exp \left(\lambda_{i} a_{i}-\frac{1}{2} P_{i j} a_{i} a_{j}\right) \exp \left(\mu_{i} a_{i}^{\dagger}-\frac{1}{2} Q_{i j} a_{i}^{\dagger} a_{j}^{\dagger}\right)\right| 0\right\rangle \\
= & \operatorname{det}(K)^{-1 / 2} \exp \left(\mu^{T} K^{-1} \lambda-\frac{1}{2} \lambda^{T} Q K^{-1} \lambda-\frac{1}{2} \mu^{T} K^{-1} P \mu\right), K \equiv 1-P Q,
\end{aligned}
$$

and setting $P=-(1-\epsilon)\left(\begin{array}{ll}1 & 0 \\ 0 & 1\end{array}\right), Q=\mathcal{V} \equiv\left(\begin{array}{ll}V^{11} & V^{12} \\ V^{21} & V^{22}\end{array}\right), \lambda=\left(\begin{array}{c}2 i E^{-1} x_{1} \\ 2 i E^{-1} x_{2}\end{array}\right)$ and $\mu=-\left(\begin{array}{cc}V^{13} & a^{(3) \dagger} \\ V^{23} & a^{(3) \dagger}\end{array}\right)$, we get:

$$
\begin{aligned}
& \left|x_{1}, \epsilon\right\rangle \star\left|x_{2}, \epsilon\right\rangle= \\
& K_{0}^{52}\left\{\operatorname{det}(1+(1-\epsilon) \mathcal{V})^{-1 / 2}\right\}^{26} \exp \left(-x_{1} \cdot E^{-2} x_{1}-x_{2} \cdot E^{-2} x_{2}\right) \times \\
& \times \exp \left\{\mu^{T} \cdot \frac{1}{1+(1-\epsilon) \mathcal{V}} \lambda-\frac{1}{2} \lambda^{T} \cdot \frac{\mathcal{V}}{1+(1-\epsilon) \mathcal{V}} \lambda+\frac{1}{2} \mu^{T} \cdot \frac{1-\epsilon}{1+(1-\epsilon) \mathcal{V}} \mu-\frac{1}{2} a^{(3) \dagger} \cdot V^{33} a^{(3) \dagger}\right\}|0\rangle .
\end{aligned}
$$

The inverse of the matrix $(1+(1-\epsilon) \mathcal{V})=\left(\begin{array}{cc}1+(1-\epsilon) C X & (1-\epsilon) C Y \\ (1-\epsilon) C Z & 1+(1-\epsilon) C X\end{array}\right)$ can be found as follows ${ }^{6}$ : From the inverse calculated numerically, we infer the following Ansatz for the inverse: $\left(\begin{array}{cc}\mu+W & -W C \\ -C W & \mu+C W C\end{array}\right)$, where $\mu$ is a real number multiplying the identity matrix and $W$ is an unknown matrix. To find $W$, we ask that the following matrix $M$ be block-diagonal:

$$
M=\left(\begin{array}{ll}
M_{11} & M_{12} \\
M_{21} & M_{22}
\end{array}\right) \equiv\left(\begin{array}{cc}
\mu+W & -W C \\
-C W & \mu+C W C
\end{array}\right) \cdot\left(\begin{array}{cc}
1+(1-\epsilon) C X & (1-\epsilon) C Y \\
(1-\epsilon) C Z & 1+(1-\epsilon) C X
\end{array}\right) .
$$

Requiring that $M_{12}=M_{21}=0$, we get $W=\mu Z\left(\frac{1}{1-\epsilon}+C X-Z\right)^{-1}$. Now substituting this expression for $W$ into (3.28), we find $M_{11}=\mu(1+(1-\epsilon) C X+(1-\epsilon) Z)$ and $M_{22}=C M_{11} C$.

\footnotetext{
${ }^{6}$ Note that the submatrices in this block-matrix do not commute with each other, making the task of finding the inverse nontrivial.
} 
The inverse is therefore $\left(\begin{array}{cc}M_{11}^{-1} & 0 \\ 0 & M_{22}^{-1}\end{array}\right) \cdot\left(\begin{array}{cc}\mu+W & -W C \\ -C W & \mu+C W C\end{array}\right)$. Namely:

$$
(1+(1-\epsilon) \mathcal{V})^{-1}=\frac{1}{1-\epsilon}\left(\begin{array}{cc}
H^{+}(\epsilon)\left(\frac{1}{1-\epsilon}+C X\right) H^{-}(\epsilon) & -H^{+}(\epsilon) Z H^{-}(\epsilon) C \\
-C H^{+}(\epsilon) Z H^{-}(\epsilon) & C H^{+}(\epsilon)\left(\frac{1}{1-\epsilon}+C X\right) H^{-}(\epsilon) C
\end{array}\right)
$$

where we have defined

$$
H^{ \pm}(\epsilon) \equiv\left(\frac{1}{1-\epsilon}+C X \pm Z\right)^{-1}
$$

The expression (3.29) was constructed by requiring that it be a left inverse of $(1+(1-\epsilon) \mathcal{V})$, but one can check that this is also a right inverse.

The matrix $H^{+}(\epsilon)$ can be expressed more explicitly in terms of the inverse of $\left(\frac{1}{1-\epsilon}-X\right)$, which behaves well because it commutes with $C, X, Y$ and $Z$. In order to find the form of this inverse, we make the following Ansatz: $H^{+}(\epsilon)=f(X)(\alpha-\beta C X+\gamma Y)$, where $\alpha, \beta$ and $\gamma$ are real numbers, and $f(X)$ is a function involving only the matrix $X$ (and the identity matrix). One can solve for all these parameters and we find:

$$
\begin{aligned}
H^{+}(\epsilon) & =\frac{1}{2-\epsilon} \frac{1}{\frac{1}{1-\epsilon}-X}(1-(1-\epsilon) C X+(1-\epsilon) Y) \\
& =\frac{1}{2} \frac{1}{1-X}(1-C X+Y)+\mathcal{O}(\epsilon) \\
& =\frac{1}{4}\left(\begin{array}{cc}
\left(1+U_{o o}\right)^{-1}\left(5-U_{o o}\right) & -\sqrt{3} i\left(1+U_{o o}\right)^{-1} U_{o e} \\
\sqrt{3} i\left(1-U_{e e}\right)^{-1} U_{e o} & 3
\end{array}\right)+\mathcal{O}(\epsilon) .
\end{aligned}
$$

In the second line, we wrote only the first term in the power expansion in $\epsilon$, and in the third line we expressed the above line in the parity notation. In the rest of the paper, we will often neglect to write the $\mathcal{O}(\epsilon)$ terms when they are not relevant.

The matrix $H^{-}(\epsilon)$ is a little bit more problematic. It can be seen to be

$$
H^{-}(\epsilon)=-\frac{1}{\epsilon} \frac{1}{\frac{1}{1-\epsilon}+X}(-1+(1-\epsilon) C X+(1-\epsilon) Y) .
$$

We see that it is singular when $\epsilon=0$; this is precisely why we have to use the regulator $\epsilon$. Let us define the singular and regular parts of $H^{-}(\epsilon)$ in the following way:

$$
H^{-}(\epsilon) \equiv \frac{1}{\epsilon} H_{\text {sing }}^{-}+H_{\text {reg }}^{-}+\mathcal{O}(\epsilon)
$$

where

$$
H_{\text {sing }}^{-}=\frac{1}{1+X}(1-C X-Y)
$$




$$
\begin{aligned}
& =\frac{1}{1+X}\left(\begin{array}{cc}
1-U_{o o} & \frac{\sqrt{3}}{3} i U_{o e} \\
-\frac{\sqrt{3}}{3} i U_{e o} & \frac{1}{3}-\frac{1}{3} U_{e e}
\end{array}\right), \\
H_{\mathrm{reg}}^{-} & =\frac{1}{(1+X)^{2}}\left(-1+2 Y+X Y+C\left(2 X+X^{2}\right)\right) \\
& =\frac{1}{(1+X)^{2}}\left(\begin{array}{cc}
-1+\frac{7}{3} U_{o o}-\frac{2}{3} U_{o o}^{2} & -\frac{7}{9} \sqrt{3} i U_{o e}+\frac{2}{9} \sqrt{3} i U_{o o} U_{o e} \\
\frac{7}{9} \sqrt{3} i U_{e o}+\frac{2}{9} \sqrt{3} i U_{e e} U_{e o} & \frac{5}{9}+\frac{11}{9} U_{e e}+\frac{2}{9} U_{e e}^{2}
\end{array}\right) .
\end{aligned}
$$

With these expressions in hand, one can now calculate explicitly the star-product (3.27).

\section{Technical details of the calculation}

To begin, let us write

$$
\left|x_{1}, \epsilon\right\rangle \star\left|x_{2}, \epsilon\right\rangle=K_{0}^{52}\left(\operatorname{det}(1+(1-\epsilon) \mathcal{V})^{-\frac{1}{2}}\right)^{26} \Omega\left(x_{1}, x_{2}\right) e^{\ell\left(a^{\dagger}, x_{1}, x_{2}\right)} e^{\frac{1}{2} a^{\dagger} \cdot \Gamma a^{\dagger}}|0\rangle,
$$

where $\Omega$ is the part of (3.27) quadratic in $x, e^{\ell}$ is the part linear in $x$, and $\Gamma$ doesn't depend on $x$.

We will start by calculating $\Gamma$ in subsection 1.1, in the next subsection we will calculate the terms

proportional to $\frac{1}{\epsilon}$ in $\Omega$, leaving the regular terms in $\Omega$ for subsection 4.3 . Then in subsection 4.4, we will calculate the linear term $\ell$. And finally we will look at the overall normalization in subsection 4.5, and we will derive the form of the star-product in the wavefunction representation.

\subsection{Term quadratic in $a^{\dagger}$}

Our goal here is to prove that $\Gamma=1$.

From (3.27), we can write

$$
\begin{aligned}
\Gamma & =\left(\begin{array}{ll}
V^{31} & V^{32}
\end{array}\right) \frac{1-\epsilon}{1+(1-\epsilon) \mathcal{V}}\left(\begin{array}{c}
V^{13} \\
V^{23}
\end{array}\right)-V^{33} \\
& =\left(\begin{array}{ll}
C Y & C Z
\end{array}\right) \frac{1-\epsilon}{1+(1-\epsilon) \mathcal{V}}\left(\begin{array}{c}
C Z \\
C Y
\end{array}\right)-C X \\
& =(\Lambda+C \Lambda C)-C X,
\end{aligned}
$$

where

$$
\Lambda \equiv Y H^{+}(\epsilon)\left(\frac{1}{1-\epsilon}+C X\right) H^{-}(\epsilon) Y-Y H^{+}(\epsilon) Z H^{-}(\epsilon) Y C .
$$


From (4.2), it is clear that $C \Gamma C=\Gamma$, which implies that $\Gamma_{o e}=\Gamma_{e o}=0$; and also $\Gamma_{o o}=2 \Lambda_{o o}+X_{o o}$ and $\Gamma_{e e}=2 \Lambda_{e e}-X_{e e}$. From (4.3), we see that

$$
\begin{aligned}
\Lambda_{o o} & =\left\{Y\left(\frac{1}{1-\epsilon}+C X+Z\right)^{-1}\left(\frac{1}{1-\epsilon}+C X+Z\right)\left(\frac{1}{1-\epsilon}+C X-Z\right)^{-1} Y\right\}_{o o} \\
& =\left\{Y H^{-}(\epsilon) Y\right\}_{o o}, \\
\Lambda_{e e} & =\left\{Y\left(\frac{1}{1-\epsilon}+C X+Z\right)^{-1}\left(\frac{1}{1-\epsilon}+C X-Z\right)\left(\frac{1}{1-\epsilon}+C X-Z\right)^{-1} Y\right\}_{e e} \\
& =\left\{Y H^{+}(\epsilon) Y\right\}_{e e} .
\end{aligned}
$$

We will now proceed further by using the parity notation. Let us first calculate $\Lambda_{e e}$ (denoting by “..." matrix elements that we don't need to know):

$$
\begin{aligned}
& \Lambda_{e e}=\left\{Y H^{+}(\epsilon) Y\right\}_{e e} \\
& =\left\{\frac{1}{3 \cdot 4}\left(\begin{array}{cc}
1+U_{o o} & -\sqrt{3} i U_{o e} \\
\sqrt{3} i U_{e o} & 1-U_{e e}
\end{array}\right)\left(\begin{array}{cc}
\left(1+U_{o o}\right)^{-1}\left(5-U_{o o}\right) & -\sqrt{3} i\left(1+U_{o o}\right)^{-1} U_{o e} \\
\sqrt{3} i\left(1-U_{e e}\right)^{-1} U_{e o} & 3
\end{array}\right) Y\right\}_{e e} \\
& =\left\{\frac{1}{3 \cdot 4 \cdot 3}\left(\begin{array}{cc}
\cdots & \cdots \\
6 \sqrt{3} i U_{e o}\left(1+U_{o o}\right)^{-1} & 6
\end{array}\right)\left(\begin{array}{cc}
\cdots & -\sqrt{3} i U_{o e} \\
\cdots & 1-U_{e e}
\end{array}\right)\right\}_{e e} \\
& =\frac{1}{3}\left(2+U_{e e}\right) \text {, }
\end{aligned}
$$

and thus

$$
\Gamma_{e e}=\frac{2}{3}\left(2+U_{e e}\right)-X_{e e}=\frac{2}{3}\left(2+U_{e e}\right)-\frac{1}{3}\left(1+2 U_{e e}\right)=1 .
$$

Let us now turn to $\Lambda_{o o}$. Its singular part is

$$
\begin{aligned}
\left(\Gamma_{o o}\right)_{\text {sing }} & =\left\{Y H_{\text {sing }}^{-} Y\right\}_{o o} \\
& =\left\{\frac{1}{3(1+X)}\left(\begin{array}{cc}
1+U_{o o} & -\sqrt{3} i U_{o e} \\
\sqrt{3} i U_{e o} & 1-U_{e e}
\end{array}\right)\left(\begin{array}{cc}
1-U_{o o} & \frac{\sqrt{3}}{3} i U_{o e} \\
-\frac{\sqrt{3}}{3} i U_{e o} & \frac{1}{3}-\frac{1}{3} U_{e e}
\end{array}\right) Y\right\}_{o o} \\
& =\left\{\frac{1}{3(1+X)}\left(\begin{array}{cc}
0 & 0 \\
\ldots & \ldots
\end{array}\right) Y\right\}_{o o}=0,
\end{aligned}
$$

and its regular part is

$$
\left(\Gamma_{o o}\right)_{\text {reg }}=\left\{Y H_{\text {reg }}^{-} Y\right\}_{o o}=
$$




$$
\begin{aligned}
& =\left\{\frac{1}{3(1+X)^{2}}\left(\begin{array}{cc}
1+U_{o o} & -\sqrt{3} i U_{o e} \\
\sqrt{3} i U_{e o} & 1-U_{e e}
\end{array}\right)\left(\begin{array}{cc}
-1+\frac{7}{3} U_{o o}-\frac{2}{3} U_{o o}^{2} & -\frac{7}{9} \sqrt{3} i U_{o e}+\frac{2}{9} \sqrt{3} i U_{o o} U_{o e} \\
\frac{7}{9} \sqrt{3} i U_{e o}+\frac{2}{9} \sqrt{3} i U_{e e} U_{e o} & \frac{5}{9}+\frac{11}{9} U_{e e}+\frac{2}{9} U_{e e}^{2}
\end{array}\right) Y\right\}_{o o} \\
& =\left\{\frac{1}{27(1+X)^{2}}\left(\begin{array}{cc}
4+2 U_{o o}-2 U_{o o}^{2} & -4 \sqrt{3} i U_{o e}+2 \sqrt{3} i U_{o o} U_{o e} \\
\ldots & \ldots
\end{array}\right)\left(\begin{array}{cc}
1+U_{o o} & \cdots \\
\sqrt{3} i U_{e o} & \ldots
\end{array}\right)\right\}_{o o} \\
& =\frac{1}{27}\left\{\frac{1}{(1+X)^{2}}\right\}_{o o}\left(16-12 U_{o o}^{2}+4 U_{o o}^{3}\right) \\
& =\frac{1}{3}+\frac{1}{3} U_{o o} .
\end{aligned}
$$

Thus $\Gamma_{o o}=\frac{2}{3}+\frac{2}{3} U_{o o}+\left(\frac{1}{3}-\frac{2}{3} U_{o o}\right)=1$, which concludes the proof that $\Gamma=1$.

\subsection{The delta function}

Now that the reader is used to the formalism, we can track down the delta function that we expect.

We will see that it is of the form

$$
\lim _{\substack{\epsilon \rightarrow 0 \\ \epsilon>0}}\left(\operatorname{det}\left(\frac{1}{\pi \epsilon} M\right)^{\frac{1}{2}} e^{-\frac{1}{\epsilon} y \cdot M y}\right)=\delta(y),
$$

where $M$ is strictly positive definite. For this, we will concentrate on the terms proportional to $\frac{1}{\epsilon}$ appearing in the exponential of (3.27). We have already shown that such terms don't appear in the term quadratic in $a^{\dagger}$ and we will show later that they neither appear in the term linear in $a^{\dagger}$.

For now let us calculate the term quadratic in $x$. From (3.27) this is

$$
\begin{aligned}
\Omega & \equiv \exp \left\{-x_{1} \cdot E^{-2} x_{1}-x_{2} \cdot E^{-2} x_{2}+2\left(x_{1} \cdot E^{-1}, x_{2} \cdot E^{-1}\right)\left(\frac{\mathcal{V}}{1+(1-\epsilon) \mathcal{V}}\right)\left(\begin{array}{c}
E^{-1} x_{1} \\
E^{-1} x_{2}
\end{array}\right)\right\} \\
& =\exp \left\{\left(x_{1} \cdot E^{-1}, x_{2} \cdot E^{-1}\right) W\left(\begin{array}{c}
E^{-1} x_{1} \\
E^{-1} x_{2}
\end{array}\right)\right\},
\end{aligned}
$$

where

$$
W \equiv(1+2 \epsilon)\left(1-2 \frac{1-\epsilon}{1+(1-\epsilon) \mathcal{V}}\right)+\mathcal{O}(\epsilon) .
$$

Again, we separate $\Omega$ and $W$ into a regular and a singular part: $\Omega=\Omega_{\text {reg }} \Omega_{\text {sing }}, W=W_{\text {reg }}+\frac{1}{\epsilon} W_{\text {sing }}$, with

$$
\begin{aligned}
& \Omega_{\text {reg }}=\exp \left\{\left(x_{1} \cdot E^{-1}, x_{2} \cdot E^{-1}\right) W_{\text {reg }}\left(\begin{array}{c}
E^{-1} x_{1} \\
E^{-1} x_{2}
\end{array}\right)\right\}, \\
& \Omega_{\text {sing }}=\exp \left\{\left(x_{1} \cdot E^{-1}, x_{2} \cdot E^{-1}\right) \frac{1}{\epsilon} W_{\text {sing }}\left(\begin{array}{c}
E^{-1} x_{1} \\
E^{-1} x_{2}
\end{array}\right)\right\} .
\end{aligned}
$$


We will come back later to the regular part, and we focus now on the singular piece

$$
\begin{aligned}
& W_{\text {sing }}=\left(\begin{array}{cc}
\left(W_{\text {sing }}\right)_{11} & \left(W_{\text {sing }}\right)_{12} \\
C\left(W_{\text {sing }}\right)_{12} C & C\left(W_{\text {sing }}\right)_{11} C
\end{array}\right), \\
& \left(W_{\text {sing }}\right)_{11}=-2\left[H^{+}(\epsilon)\left(\frac{1}{1-\epsilon}+C X\right) H_{\text {sing }}^{-}\right]_{\epsilon=0}, \quad\left(W_{\text {sing }}\right)_{12}=2\left[H^{+}(\epsilon) Z H_{\text {sing }}^{-}\right]_{\epsilon=0} C
\end{aligned}
$$

In order to calculate $\Omega_{\text {sing }}$, we will need the following identities:

$$
\begin{aligned}
H^{+}(0) Z H_{\text {sing }}^{-} & =\frac{1}{2} H_{\text {sing }}^{-}, \\
H^{+}(0)(1+C X) H_{\text {sing }}^{-} & =\frac{1}{2} H_{\text {sing }}^{-}, \\
E^{-1} A^{+} \tilde{A}^{+} E & =\frac{1}{2}\left(\begin{array}{ccc}
1 & E^{-1}\left(\Upsilon_{e o}\right)^{-1} L & E \\
E^{-1} \Upsilon_{e o} E & 1 & 1
\end{array}\right) \\
& =\frac{1}{2}\left(\begin{array}{ccc}
\sqrt{3} i\left(1-U_{e e}\right)^{-1} U_{e o} & -\frac{i}{\sqrt{3}}\left(1-U_{o o}\right)^{-1} U_{o e} \\
1
\end{array}\right), \\
H_{\text {sing }}^{-} E^{-1} A^{+} & =0 .
\end{aligned}
$$

The first one can be shown straightforwardly in the parity notation, the second one follows from the first one, and the third one can be established using the definitions of $A^{ \pm}, \tilde{A}^{ \pm}$and $\Upsilon$ as well as (2.30) and (2.31); $\left(\Upsilon_{e o}\right)^{-1_{L}}$ stands for the left inverse of $\Upsilon_{e o}$. Finally, to show the last identity, one can show in the parity notation, using (4.12), that $H_{\text {sing }}^{-} E^{-1} A^{+} \tilde{A}^{+} E=0$. The identity then follows because $\tilde{A}^{+}$is invertible on the right.

Let us now calculate the term, in the exponential of $\Omega_{\text {sing }}$, quadratic in $x_{1}$ :

$$
\begin{aligned}
x_{1} \cdot E^{-1}\left(W_{\text {sing }}\right)_{11} E^{-1} x_{1} & =-2 x_{1} \cdot E^{-1} H^{+}(0)(1+C X) H_{\text {sing }}^{-} E^{-1} x_{1} \\
& =-x_{1} \cdot E^{-1} H_{\text {sing }}^{-} E^{-1} x_{1} \\
& =-x_{1} \cdot E^{-1} H_{\text {sing }}^{-} E^{-1}\left(A^{+} x_{1}^{L}+A^{-} x_{1}^{R}\right) \\
& =-x_{1} \cdot E^{-1} H_{\text {sing }}^{-} E^{-1} A^{-} x_{1}^{R} \\
& =-x_{1}^{R} \cdot\left(A^{-}\right)^{T} E^{-1} H_{\text {sing }}^{-} E^{-1}\left(A^{+} x_{1}^{L}+A^{-} x_{1}^{R}\right) \\
& =-x_{1}^{R} \cdot\left(A^{-}\right)^{T} E^{-1} H_{\text {sing }}^{-} E^{-1} A^{-} x_{1}^{R} \\
& =-x_{1}^{R} \cdot M x_{1}^{R},
\end{aligned}
$$

where

$$
M \equiv\left(A^{-}\right)^{T} E^{-1} H_{\text {sing }}^{-} E^{-1} A^{-} .
$$


The term quadratic in $x_{2}$ is calculated similarly:

$$
\begin{aligned}
x_{2} \cdot E^{-1}\left(W_{\text {sing }}\right)_{22} E^{-1} x_{2} & =-x_{2} \cdot E^{-1} C H_{\text {sing }}^{-} C E^{-1} x_{2} \\
& =-x_{2} \cdot E^{-1} C H_{\text {sing }}^{-} E^{-1} C\left(A^{+} x_{2}^{L}+A^{-} x_{2}^{R}\right) \\
& =-x_{2} \cdot E^{-1} C H_{\text {sing }}^{-} E^{-1}\left(A^{-} x_{2}^{L}+A^{+} x_{2}^{R}\right) \\
& =-x_{2} \cdot C E^{-1} H_{\text {sing }}^{-} E^{-1} A^{-} x_{2}^{L} \\
& =-x_{2}^{L} \cdot M x_{2}^{L} .
\end{aligned}
$$

The calculation of the terms containing $x_{1}$ and $x_{2}$ is similar to the above ones and we can see that $\Omega_{\text {sing }}$ can be written

$$
\Omega_{\text {sing }}=\exp \left(-\frac{1}{\epsilon}\left(x_{1}^{R}-x_{2}^{L}\right) \cdot M\left(x_{1}^{R}-x_{2}^{L}\right)\right) .
$$

We can see that $M$ is positive definite by writing $M=B^{T} B$, where

$$
B=\frac{1}{\sqrt{2}}(1+X)^{-1 / 2}(1+C)(1-Y)^{1 / 2} E^{-1} A^{-} .
$$

To verify this, the reader should keep in mind that $\left(X^{2}\right)^{1 / 2}=-X$ if the square root is defined to have only positive eigenvalues. Indeed we assumed that the spectrum of $X$ is contained in the interval $\left[-\frac{1}{3}, 0\right]$. Though there is still no proof of this, there is compelling numerical evidence that it is indeed true ${ }^{7}$.

Unfortunately, $M$ doesn't seem to be strictly positive definite since $(1+C)$ is singular; we thus expect $B$, and therefore $M$, to have some zero eigenvalues. Numerical analysis shows that this might not be the case: If we truncate all matrices to a size $N$, we find indeed $N / 2$ "small" eigenvalues, but the corresponding normalized eigenvectors all have some "non-small" components which have indices $\geq N / 2$. Therefore we believe that, when we take the limit $N \rightarrow \infty$, these eigenvectors cannot be normalized anymore, and thus disappear from the spectrum. A better understanding of this phenomenon would be interesting to pursue.

For now, we will assume that $M$ is strictly positive definite and thus, if we restrict $\epsilon$ to be positive, (4.15) gives, in the limit $\epsilon \rightarrow 0$ :

$$
\Omega_{\text {sing }}=\operatorname{det}\left(\frac{1}{\pi \epsilon} M\right)^{-\frac{1}{2}} \delta\left(x_{1}^{R}-x_{2}^{L}\right),
$$

which was expected if we think of the star-product to glue the right-half of the first string to the left-half of the second string.

\footnotetext{
${ }^{7}$ I thank B. Zwiebach for a discussion of this point
} 


\subsection{Regular quadratic term in $x$}

We now wish to calculate $\Omega_{\text {reg }}$ (4.7). For this, we first note that $W_{\text {reg }}$ gets contributions from two different kinds of terms: We will write $W_{\text {reg }}=W_{\text {reg }}^{(1)}+W_{\text {reg }}^{(2)}$, where $W_{\text {reg }}^{(1)}$ denotes the terms that are a product of a term proportional to $\epsilon$ and a singular term (proportional to $\frac{1}{\epsilon}$ ) coming from the singular part of $\mathrm{H}^{-}(\epsilon) ; W_{\text {reg }}^{(2)}$ denotes all the other terms.

Let us first calculate the contribution from $W_{\text {reg }}^{(1)}$ in $\Omega_{\text {reg }}$. As defined above, $W_{\text {reg }}^{(1)}$ can be expressed from (4.8) and (4.9) by keeping the terms proportional to $\epsilon$ that are multiplying $H_{\text {sing. }}^{-}$. These are:

$$
\begin{aligned}
W_{\text {reg }}^{(1)} & =\left(\begin{array}{cc}
\left(W_{\text {reg }}^{(1)}\right)_{11} & \left(W_{\text {reg }}^{(1)}\right)_{12} \\
C\left(W_{\text {reg }}^{(1)}\right)_{12} C & C\left(W_{\text {reg }}^{(1)}\right)_{11} C
\end{array}\right), \\
\left(W_{\text {reg }}^{(1)}\right)_{11} & =2\left(W_{\text {sing }}\right)_{11}-2\left[\frac{d}{d \epsilon}\left(H^{+}(\epsilon)\left(\frac{1}{1-\epsilon}+C X\right)\right)\right]_{\epsilon=0} H_{\text {sing }}^{-}+\mathcal{O}(\epsilon) \\
& =2\left(W_{\text {sing }}\right)_{11}-2 H^{+}(0)^{2} Z H_{\text {sing }}^{-}+\mathcal{O}(\epsilon) \\
& =2\left(W_{\text {sing }}\right)_{11}-H^{+}(0) H_{\text {sing }}^{-}+\mathcal{O}(\epsilon), \\
\left(W_{\text {reg }}^{(1)}\right)_{12} & =2\left(W_{\text {sing }}\right)_{12}+2\left[\frac{d}{d \epsilon}\left(H^{+}(\epsilon) Z\right)\right]_{\epsilon=0} H_{\text {sing }}^{-} C+\mathcal{O}(\epsilon) \\
& =2\left(W_{\text {sing }}\right)_{12}-H^{+}(0) H_{\text {sing }}^{-} C+\mathcal{O}(\epsilon),
\end{aligned}
$$

where we have used (4.10). After substituting $x=A^{+} x^{L}+A^{-} x^{R}$ in (4.7) and using $x_{1}^{R}=x_{2}^{L}$, it is straightforward to see that the $W_{\text {sing }}$ terms in (4.18) will disappear, leaving us with

$$
\begin{aligned}
& \left(x_{1} \cdot E^{-1}, x_{2} \cdot E^{-1}\right) W_{\mathrm{reg}}^{(1)}\left(\begin{array}{c}
E^{-1} x_{1} \\
E^{-1} x_{2}
\end{array}\right)= \\
& =-\left(x_{1} \cdot E^{-1}, x_{2} \cdot E^{-1}\right)\left(\begin{array}{cc}
H^{+}(0) H_{\text {sing }}^{-} & H^{+}(0) H_{\text {sing }}^{-} C \\
C H^{+}(0) H_{\text {sing }}^{-} & C H^{+}(0) H_{\text {sing }}^{-} C
\end{array}\right)\left(\begin{array}{c}
E^{-1} A^{+} x_{1}^{L}+E^{-1} A^{-} x_{2}^{L} \\
E^{-1} A^{+} x_{2}^{L}+E^{-1} A^{-} x_{2}^{R}
\end{array}\right) \\
& =-2\left(x_{1}^{L} \cdot A^{+T} E^{-1}+x_{2}^{L} \cdot A^{-T} E^{-1}, x_{2}^{L} \cdot A^{+T} E^{-1}+x_{2}^{R} \cdot A^{-T} E^{-1}\right)\left(\begin{array}{c}
H^{+}(0) H_{\text {sing }}^{-} E^{-1} A^{-} x_{2}^{L} \\
C H^{+}(0) H_{\text {sing }}^{-} E^{-1} A^{-} x_{2}^{L}
\end{array}\right),
\end{aligned}
$$

where we have made use of (4.13).

We now turn to $W_{\text {reg }}^{(2)}$. We have:

$$
W_{\text {reg }}^{(2)}=\left(\begin{array}{cc}
\left(W_{\text {reg }}^{(2)}\right)_{11} & \left(W_{\text {reg }}^{(2)}\right)_{12} \\
C\left(W_{\text {reg }}^{(2)}\right)_{12} C & C\left(W_{\text {reg }}^{(2)}\right)_{11} C
\end{array}\right),
$$




$$
\begin{aligned}
& \left(W_{\text {reg }}^{(2)}\right)_{11}=1-2 H^{+}(0)(1+C X) H_{\text {reg }}^{-} \\
& \left(W_{\text {reg }}^{(2)}\right)_{12}=2 H^{+}(0) Z H_{\text {reg }}^{-} C .
\end{aligned}
$$

We will need the following identities:

$$
\begin{aligned}
& A^{+T} E^{-1}\left(W_{\text {reg }}^{(2)}\right)_{11} E^{-1} A^{+}=-A^{+T} E^{-2} A^{+}, \\
& A^{+T} E^{-1}\left(\left(W_{\text {reg }}^{(2)}\right)_{12}+C\left(W_{\text {reg }}^{(2)}\right)_{12}^{T} C\right) E^{-1} A^{-}=-2 A^{+T} E^{-2} A^{-}, \\
& H^{+}(0) E^{-1} A^{-}=\frac{1}{2} E^{-1} A^{-}, \\
& A^{-T} E^{-1} H^{+}(0) H_{\text {sing }}^{-} E^{-1} A^{-}=A^{-T} E^{-1}\left(\frac{1}{2}-H^{+}(0)(1+C X-Z) H_{\text {reg }}^{-}\right) E^{-1} A^{-} .
\end{aligned}
$$

Equations (4.23) - (4.25) can be established straightforwardly with the parity notation (Note that to use the parity notation, one must first multiply 4.23 on the left by $E \tilde{A}^{+T}$ and on the right by $\tilde{A}^{+} E$, and also multiply (4.24) on the left by $E \tilde{A}^{+T}$ and on the right by $\tilde{A}^{-} E$. This is allowed since $\tilde{A}^{ \pm}$have a right inverse and thus $\tilde{A}^{ \pm T}$ have a left inverse). Equation (4.26) can be proved

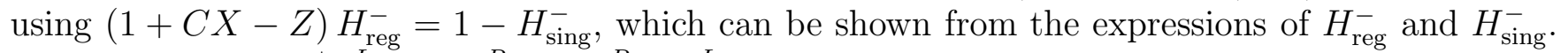
Substituting $x=A^{+} x^{L}+A^{-} x^{R}$ and $x_{1}^{R}=x_{2}^{L}$, we get

$$
\begin{aligned}
& \left(x_{1} \cdot E^{-1}, x_{2} \cdot E^{-1}\right) W_{\mathrm{reg}}^{(2)}\left(\begin{array}{c}
E^{-1} x_{1} \\
E^{-1} x_{2}
\end{array}\right)= \\
& =\left(x_{1}^{L} \cdot A^{+T}+x_{2}^{L} \cdot A^{-T}, x_{2}^{L} \cdot A^{+T}+x_{2}^{R} \cdot A^{-T}\right) E^{-1} W_{\mathrm{reg}}^{(2)} E^{-1}\left(\begin{array}{c}
A^{+} x_{1}^{L}+A^{-} x_{2}^{L} \\
A^{+} x_{2}^{L}+A^{-} x_{2}^{R}
\end{array}\right) .
\end{aligned}
$$

Using (4.23) - (4.26), it is straightforward to calculate each term in (4.19) and (4.27). And as a final result, we get, as was expected:

$$
\begin{aligned}
\Omega_{\mathrm{reg}} & =\exp \left(\left(x_{1} \cdot E^{-1}, x_{2} \cdot E^{-1}\right) W_{\mathrm{reg}}^{(1)}\left(\begin{array}{c}
E^{-1} x_{1} \\
E^{-1} x_{2}
\end{array}\right)+\left(x_{1} \cdot E^{-1}, x_{2} \cdot E^{-1}\right) W_{\mathrm{reg}}^{(2)}\left(\begin{array}{c}
E^{-1} x_{1} \\
E^{-1} x_{2}
\end{array}\right)\right) \\
& =\exp \left(-\left(A^{+} x_{1}^{L}+A^{-} x_{2}^{R}\right) \cdot E^{-2}\left(A^{+} x_{1}^{L}+A^{-} x_{2}^{R}\right)\right) .
\end{aligned}
$$

\subsection{Term linear in $a^{\dagger}$}

From (3.27), we see that the term linear in $a^{\dagger}$ is

$$
\begin{aligned}
\ell & =-2 i a^{\dagger} \cdot\left(\left(V_{12} D_{11}+V_{21} D_{21}\right) E^{-1} x_{1}+\left(V_{12} D_{12}+V_{21} D_{22}\right) E^{-1} x_{2}\right), D \equiv\left(\frac{1}{1+(1-\epsilon) \mathcal{V}}\right) \\
& =-2 i a^{\dagger} \cdot\left(\left(V_{12} D_{11}+V_{21} D_{21}\right) E^{-1} x_{1}+C\left(V_{12} D_{11}+V_{21} D_{21}\right) C E^{-1} x_{2}\right) .
\end{aligned}
$$


We have

$$
\begin{aligned}
V_{12} D_{11}+V_{21} D_{21} & =C Y D_{11}+Y C D_{21} \\
& =\frac{1}{1-\epsilon}\left(C Y H^{+}(\epsilon)\left(\frac{1}{1-\epsilon}+C X\right) H^{-}(\epsilon)-Y H^{+}(\epsilon) Z H^{-}(\epsilon)\right) .
\end{aligned}
$$

Using (4.10) and (4.11), and noting that $\left(Y H_{\text {sing }}^{-}\right)_{o o}=\left(Y H_{\text {sing }}^{-}\right)_{o e}=0$, we see that the singular terms don't contribute in $\ell$. it is then straightforward to show, using (4.12), that to zeroth order in $\epsilon$ we have

$$
\begin{aligned}
& V_{12} D_{11}+V_{21} D_{21}=\left(\begin{array}{cc}
-\frac{1+U_{o o}}{4-2 U_{o o}} & \sqrt{3} i\left(4-2 U_{o o}\right)^{-1} U_{o e} \\
\frac{1}{2} E^{-1} \Upsilon_{e o} E & \frac{1}{2}
\end{array}\right)= \\
= & C E^{-1}\left(\begin{array}{cc}
\frac{1}{2} & \frac{1}{2}\left(\Upsilon_{e o}\right)^{-1}{ }^{-1} \\
\frac{1}{2} \Upsilon_{e o} & \frac{1}{2}
\end{array}\right) E+C E^{-1}\left(\begin{array}{cc}
-E \frac{1-2 U_{o o}}{2-U_{o o}} E & 0 \\
0 & 0
\end{array}\right)\left(\begin{array}{cc}
\frac{1}{2} & -\frac{1}{2}\left(\Upsilon_{e o}\right)^{-1_{L}} \\
-\frac{1}{2} \Upsilon_{e o} & \frac{1}{2}
\end{array}\right) E \\
= & C E^{-1} A^{+} \tilde{A}^{+} E+C E^{-1} \Theta A^{-} \tilde{A}^{-} E, \quad \Theta \equiv\left(\begin{array}{cc}
-E \frac{1-2 U_{o o}}{2-U_{o o}} E & 0 \\
0 & 0
\end{array}\right),
\end{aligned}
$$

and thus

$$
C\left(V_{12} D_{11}+V_{21} D_{21}\right) C=C E^{-1} A^{-} \tilde{A}^{-} E+C E^{-1} \Theta A^{+} \tilde{A}^{+} E .
$$

Substituting (4.31) and (4.32) into (4.29), we get

$$
\begin{aligned}
\ell & =-2 i a^{\dagger} \cdot C\left(E^{-1} A^{+} x_{1}^{L}+E^{-1} \Theta A^{-} x_{1}^{R}+E^{-1} A^{-} x_{2}^{R}+E^{-1} \Theta A^{+} x_{2}^{L}\right) \\
& =-2 i a^{\dagger} \cdot C E^{-1}\left(A^{+} x_{1}^{L}+A^{-} X_{2}^{R}+\Theta A^{-}\left(x_{1}^{R}-x_{2}^{L}\right)\right) \\
& =-2 i a^{\dagger} \cdot C E^{-1}\left(A^{+} x_{1}^{L}+A^{-} X_{2}^{R}\right)
\end{aligned}
$$

where in the second line we used $\Theta A^{+}=\Theta C C A^{+}=-\Theta A^{-}$, and in the third line we used the fact that the delta function forces $\left(x_{1}^{R}-x_{2}^{L}\right)$ to be zero.

\subsection{The overall normalization}

It is now time to pause and look at what we have until now:

$$
\left|x_{1}\right\rangle \star\left|x_{2}\right\rangle=\mathcal{K}^{26} \delta\left(x_{1}^{R}-x_{2}^{L}\right)\left|A^{+} x_{1}^{L}+A^{-} x_{2}^{R}\right\rangle,
$$

where

$$
\mathcal{K} \equiv \lim _{\epsilon \rightarrow 0}\left(K_{0} \operatorname{det}(1+(1-\epsilon) \mathcal{V})^{-\frac{1}{2}} \operatorname{det}\left(\frac{1}{\pi \epsilon} M\right)^{-1 / 2}\right)
$$


and $M$ is given by (4.14). We will now try to calculate $\mathcal{K}$ more explicitly. First note that the determinants appearing in $\mathcal{K}$ are not finite. To calculate them we use level truncation and keep oscillators of level $\leq N$ only, taking the limit $N \rightarrow \infty$ at the end. First, we have

$$
\operatorname{det}\left(\frac{1}{\pi \epsilon} M\right)^{-1 / 2}=\operatorname{det}(M)^{-\frac{1}{2}} \epsilon^{\frac{N}{2}} \pi^{\frac{N}{2}} .
$$

Now note that $(1+(1-\epsilon) \mathcal{V})^{-1}$ is a block matrix (of size $2 N$ ), but we can express its determinant in terms of the determinant of a $N$ by $N$ matrix:

$$
\begin{aligned}
(1-\epsilon)^{2 N} \operatorname{det}(1+(1-\epsilon) \mathcal{V})^{-1} & =\operatorname{det}\left(\begin{array}{cc}
H^{+}(\epsilon)\left(\frac{1}{1-\epsilon}+C X\right) H^{-}(\epsilon) & -H^{+}(\epsilon) Z H^{-}(\epsilon) C \\
-C H^{+}(\epsilon) Z H^{-}(\epsilon) & C H^{+}(\epsilon)\left(\frac{1}{1-\epsilon}+C X\right) H^{-}(\epsilon) C
\end{array}\right) \\
& =\operatorname{det}\left(\begin{array}{cc}
H^{+}(\epsilon) & -H^{+}(\epsilon) Z H^{-}(\epsilon) C \\
C H^{+}(\epsilon) & C H^{+}(\epsilon)\left(\frac{1}{1-\epsilon}+C X\right) H^{-}(\epsilon) C
\end{array}\right) \\
& =\operatorname{det}\left(\begin{array}{cc}
H^{+}(\epsilon) & -H^{+}(\epsilon) Z H^{-}(\epsilon) C \\
0 & C H^{-}(\epsilon) C
\end{array}\right) \\
& =\operatorname{det}\left(H^{+}(\epsilon)\right) \operatorname{det}\left(C H^{-}(\epsilon) C\right) \\
& \stackrel{\epsilon \rightarrow 0}{\longrightarrow} \operatorname{det}\left(H^{+}(0)\right) \operatorname{det}\left(H_{\text {sing }}^{-}\right) \epsilon^{-N},
\end{aligned}
$$

where, in the second line we added, to the first $N$ columns, the last $N$ columns multiplied on the right by $C$; this operation doesn't change the determinant. Neither does the second operation: we subtracted the $N$ first lines, multiplied by $C$ on the left, to the $N$ last lines. This set to zero the lower-left block matrix, and the determinant then becomes the product of the determinant of the upper-left block times the determinant of the lower-right block. In the last line we took the limit $\epsilon \rightarrow 0$ before the limit $N \rightarrow \infty$. We can now write

$$
\mathcal{K}=K_{0} \operatorname{det}\left(H^{+}(0)\right)^{\frac{1}{2}} \operatorname{det}(E) \operatorname{det}\left(A^{-}\right)^{-1} \pi^{\frac{N}{2}} .
$$

We see that the $\epsilon$ 's canceled each other, proving that our regularization scheme is consistent.

This normalization constant $\mathcal{K}$ plays a role when we want to express the star-product in the space of wavefunctions $\psi\left(x^{L}, x^{R}\right)$. We define the wavefunction of the state $|\psi\rangle$ by: $\psi\left(x^{L}, x^{R}\right) \equiv\langle x \mid \psi\rangle$. We expect the star-product of wavefunctions to have the following form:

$$
\left(\psi_{1} \star \psi_{2}\right)\left(x^{L}, x^{R}\right)=\mathcal{Z} \int[d y] \psi_{1}\left(x^{L}, y\right) \psi_{2}\left(y, x^{R}\right),
$$

where we integrate over the half-string $y$. In [12], $\mathcal{Z}$ was absorbed in the integration measure ${ }^{8}$ $[d y]$, but here we want to define the measure in the obvious way: $[d y]=\prod d y_{n}$, as a product over all half-string mode measures. And similarly for the path integral over whole-string modes: $[d x]=\prod d x_{n}$.

\footnotetext{
${ }^{8}$ I thank B. Zwiebach for a discussion about this point
} 
In order to formally prove (4.39) we calculate $\left|\psi_{1}\right\rangle \star\left|\psi_{2}\right\rangle$ by inserting two complete sets of zeromomentum states. And we will need to write $[d x]=J\left[d x^{L}\right]\left[d x^{R}\right]$, where $J$ is some constant relating the measures $[d x]$ and $\left[d x^{L}\right]\left[d x^{R}\right]$.

$$
\begin{aligned}
\left|\psi_{1}\right\rangle \star\left|\psi_{2}\right\rangle & =\left(\int\left[d x_{1}\right]\left|x_{1}\right\rangle\left\langle x_{1} \mid \psi_{1}\right\rangle\right) \star\left(\int\left[d x_{2}\right]\left|x_{2}\right\rangle\left\langle x_{2} \mid \psi_{1}\right\rangle\right) \\
& =\int\left[d x_{1}\right]\left[d x_{2}\right] \psi_{1}\left(x_{1}^{L}, x_{1}^{R}\right) \psi_{2}\left(x_{2}^{L}, x_{2}^{R}\right)\left(\left|x_{1}\right\rangle \star\left|x_{2}\right\rangle\right) \\
& =J^{2} \mathcal{K}^{26} \int\left[d x_{1}^{L}\right]\left[d x_{1}^{R}\right]\left[d x_{2}^{L}\right]\left[d x_{2}^{R}\right] \psi_{1}\left(x_{1}^{L}, x_{1}^{R}\right) \psi_{2}\left(x_{2}^{L}, x_{2}^{R}\right) \delta\left(x_{1}^{R}-x_{2}^{L}\right)\left|A^{+} x_{1}^{L}+A^{-} x_{2}^{R}\right\rangle \\
& =J^{2} \mathcal{K}^{26} \int\left[d x^{L}\right]\left[d x^{R}\right]\left(\int[d y] \psi_{1}\left(x^{L}, y\right) \psi_{2}\left(y, x^{R}\right)\right)|x\rangle \\
& =J \mathcal{K}^{26} \int[d x]\left(\int[d y] \psi_{1}\left(x^{L}, y\right) \psi_{2}\left(y, x^{R}\right)\right)|x\rangle .
\end{aligned}
$$

Therefore, if we impose the following normalization on the states $|x\rangle$ :

$$
\int[d x]|x\rangle\langle x|=1,
$$

we get

$$
\mathcal{Z}=J \mathcal{K}^{26} .
$$

We postpone to future work the exact calculation of the factors ${ }^{9} \mathcal{Z}$ or $J$.

\section{Summary}

In this summary, we would like to collect some useful formulas that were established in this paper.

\subsection{Factorization of the sliver}

$$
\begin{gathered}
A^{+T} E^{-1} \frac{1-S}{1+S} E^{-1} A^{-}=0, \\
\sqrt{3} i U_{e o}\left(1+U_{o o}\right)^{-1}=E^{-1} \Upsilon_{e o} E \\
-\sqrt{3} i\left(1-U_{o o}\right)\left(U_{e o}\right)^{-1}=E \Upsilon_{o e} E^{-1} .
\end{gathered}
$$

\footnotetext{
${ }^{9}$ It must be possible to relate these factors to the constants $\gamma_{1}, \gamma_{2}$ and $\gamma_{3}$ calculated in Appendices A.1 and A.2 of [20]. I thank W. Taylor for pointing this out.
} 


\subsection{Star-product}

If $\left|x_{1}\right\rangle$ and $\left|x_{2}\right\rangle$ are zero-momentum eigenstates of $\hat{x}$, then:

$$
\left|x_{1}\right\rangle \star\left|x_{2}\right\rangle=\mathcal{K}^{26} \delta\left(x_{1}^{R}-x_{2}^{L}\right)\left|A^{+} x_{1}^{L}+A^{-} x_{2}^{R}\right\rangle,
$$

where

$$
\mathcal{K}=K_{0} \operatorname{det}\left(H^{+}(0)\right)^{\frac{1}{2}} \operatorname{det}(E) \operatorname{det}\left(A^{-}\right)^{-1} \pi^{\frac{N}{2}} .
$$

The star-product of two zero-momentum string wavefunctions $\psi_{1}$ and $\psi_{2}$ is:

$$
\left(\psi_{1} \star \psi_{2}\right)\left(x^{L}, x^{R}\right)=J \mathcal{K}^{26} \int[d y] \psi_{1}\left(x^{L}, y\right) \psi_{2}\left(y, x^{R}\right),
$$

where $J$ is defined by

$$
[d x]=J\left[d x^{L}\right]\left[d x^{R}\right] .
$$

\subsection{Projectors on the sliver}

$$
\rho_{1}(1+S) E \tilde{A}^{+T}=0, \quad \rho_{2}(1+S) E \tilde{A}^{-T}=0 .
$$

\section{Discussion and Conclusions}

With an algebraic proof, very similar to the one in [19], we were able to show that the sliver wavefunction factorizes into a left part and a right part. This was already strongly believed to be true on both geometrical and numerical grounds, and it was moreover known to be true for the D-instanton sliver. This property is very important in generating classical solutions in Vacuum String Field Theory. And we already found an application of this factorization, that we used in proving that the matrices $\rho_{1}$ and $\rho_{2}$ are left and right projectors on the sliver.

We have performed a complete analytic calculation of the star-product of two eigenstates of $\hat{x}$ expressed in the oscillator basis, and have shown that we do get the expected result. That is: The star-product is zero unless the right-half of the first string exactly coincides with the left-half of the second string. Moreover, the product is an eigenstate of $\hat{x}$, whose left-half is the left-half of the first string, and whose right-half is the right-half of the second string. This allowed us to write the star-product between zero-momentum string wavefunctions as a path integral over a half-string, modulo a still unknown constant (which can be absorbed in the half-string integration measure).

This calculation, done with the infinite dimensional matrix representation of the Neumann coefficients was technically difficult, partly because we had to find inverses to nontrivial block-matrices and because we had to use a regulator in order to make singular matrices invertible. We hope that the techniques used here will find some other useful applications in performing exact calculations in string field theory. 
A direct extension of this work would be to generalize the calculation of the star-product of zero-momentum eigenstates of $\hat{x}$ to include nonzero-momentum eigenstates as well. Also, it would be interesting to generalize these results to the ghost sector. At last, it might be useful to be able to relate the integration measure over the whole-string modes to the measure over the half-string modes.

\section{Acknowledgements}

I would like to thank B. Zwiebach for suggesting to me some of the problems treated in this paper, as well as for many helpful discussions, and proofreadings of the manuscript. I also thank I. Ellwood, B. Feng, Y.-H. He and W. Taylor for discussions. This research was supported in part by the CTP and LNS of MIT, a Presidential Fellowship of MIT and the U.S. Department of Energy under cooperative research agreement \# DE-FC02-94ER40818.

\section{References}

[1] E. Witten, Noncommutative Geometry And String Field Theory, Nucl. Phys. B268, 253 (1986).

[2] D. J. Gross and A. Jevicki, Operator Formulation Of Interacting String Field Theory, Nucl. Phys. B283, 1 (1987).

[3] D. J. Gross and A. Jevicki, Operator Formulation Of Interacting String Field Theory. 2, Nucl. Phys. B287, 225 (1987).

[4] E. Cremmer, A. Schwimmer and C. B. Thorn, The Vertex Function In Witten's Formulation Of String Field Theory, Phys. Lett. B 179, 57 (1986).

[5] S. Samuel, The physical and ghost vertices in Witten's string field theory, Phys. Lett. B 181, 255 (1986).

[6] H. Chan and S. T. Tsou, String Theory Considered As A Local Gauge Theory Of An Extended Object, Phys. Rev. D 35, 2474 (1987); Yang-Mills formulation of interacting strings, Phys. Rev. D 39, 555 (1989).

[7] J. Bordes, H. Chan, L. Nellen and S. T. Tsou, Half String Oscillator Approach to String Field Theory, Nucl. Phys. B351, 441 (1991).

[8] A. Abdurrahman, F. Anton and J. Bordes, Half string oscillator approach to closed string field theory, Nucl. Phys. B397, 260 (1993); Half string oscillator approach to string field theory (ghost sector 2), Nucl. Phys. B411, 693 (1994).

[9] A. Abdurrahman and J. Bordes, The relationship between the comma theory and Witten's string field theory, Phys. Rev. D 58:086003 (1998).

[10] L. Rastelli, A. Sen and B. Zwiebach, String field theory around the tachyon vacuum, hep-th/0012251.

[11] L. Rastelli, A. Sen and B. Zwiebach, Classical Solutions in String Field Theory Around the Tachyon Vacuum, hep-th/0102112. 
[12] L. Rastelli, A. Sen and B. Zwiebach, Half-strings, Projectors, and Multiple D-branes in Vacuum String Field Theory, hep-th/0105058.

[13] L. Rastelli, A. Sen and B. Zwiebach, Boundary CFT construction of D-branes in vacuum string field theory, hep-th/0105168.

[14] P. Mukhopadhyay, Oscillator Representation of the BCFT Construction of D-branes in Vacuum String Field Theory, hep-th/0110136.

[15] L. Rastelli, A. Sen and B. Zwiebach, Vacuum string field theory, hep-th/0106010].

[16] L. Rastelli and B. Zwiebach, Tachyon potentials, star products and universality, JHEP 0109, 038 (2001) hep-th/0006240.

[17] V. A. Kostelecky and R. Potting, Analytical construction of a nonperturbative vacuum for the open bosonic string, Phys. Rev. D 63, 046007 (2001) hep-th/0008252.

[18] K. Furuuchi and K. Okuyama, Comma vertex and string field algebra, JHEP 09, 035 (2001) hepth/0107101].

[19] D. J. Gross and W. Taylor, Split string field theory. I, JHEP 0108, 009 (2001) hep-th/0105059.

[20] D. J. Gross and W. Taylor, Split string field theory. II, JHEP 0108, 010 (2001) hep-th/0106036.

[21] T. Kawano and K. Okuyama, Open string fields as matrices, JHEP 0106, 061 (2001) hep-th/0105129. 